\title{
Un modelo crítico-interpretativo para el estudio de las identidades laborales. Contribuciones a la investigación psicosocial sobre trabajo y subjetividad en América Latina*
}

\author{
A Critical-Interpretive Model for the Study of Labor \\ Identities. Contributions to Psychosocial Research \\ on Work and Subjectivity in Latin America
}

Recibido: marzo 4 de 2013 | Revisado: mayo 3 de 2013 | Aceptado: julio 1 de 2013

\author{
Antonio STECHER ** \\ Universidad Diego Portales, Chile
}

Doi: $10.11144 / J a v e r i a n a . U P S Y 12-4 . m c i e$

Para citar este artículo: Stecher, A. (2013). Un modelo crítico-interpretativo para el estudio de las identidades laborales. Contribuciones a la investigación psicosocial sobre trabajo y subjetividad en América Latina. Universitas Psychologica, 12(4), 1311-1324. Doi: 10.11144/Javeriana.UPSY12-4.mcie

Los resultados presentados en este estudio son parte de la Tesis Doctoral realizada para la obtención del grado de Doctor en Psicología Social en la Universidad Autónoma de Barcelona. La realización de dichos estudios fue posible gracias al apoyo de Comisión Nacional de Investigación Científica y Tecnológica del Gobierno de Chile (CONICYT), Fundación Carolina y Universidad Diego Portales.

** ResearcherID: M-3147-2013. La correspondencia dirigida a este artículo debe ir dirigida a Antonio Stecher, Facultad de Psicología, Universidad Diego Portales. Grajales 1898, Santiago, Chile. E-mail: antonio.stecher@udp.cl

\section{RESUMEN}

Se presenta un modelo basado en los aportes de las tradiciones narrativa, interaccionista simbólica y crítica, para estudiar las identidades laborales. Dicho modelo busca contribuir al desarrollo de la Psicología Social del Trabajo en América Latina, específicamente en lo que respecta a la investigación sobre las articulaciones entre trabajo y subjetividad en el nuevo capitalismo. Se propone una conceptualización de las identidades laborales que releva su carácter de producción simbólica articulada narrativamente, así como su carácter de proceso social y de emergente de interacciones sociales simbólicamente mediadas. Asimismo, se visibiliza el modo en que las identidades laborales, en tanto producto (narrativas) y en tanto proceso sociosimbólico, se configuran siempre dentro de contextos macrosociales e institucionales atravesados por relaciones de poder.

Palabras claves autor

Identidades laborales, trabajo, subjetividad, América Latina.

Palabras clave descriptores

Psicología social, identidad, Chile.

\section{A B S T R A C T}

This article presents a theoretical model for studying labor identities. It is based on the contributions of narrative, symbolic interactionism and critical traditions. This model is meant to be a contribution to the development of the Social Psychology of Work in Latin America, specifically in regard to research on relations between work and subjectivity in the context of the new capitalism. We propose a conceptualization of labor identities, which realizes its character of symbolic production narratively articulated (narrative identities), and its character of social process and emergent of social interactions symbolically mediated. Also visualizes how labor identities, as product (narrative) and as socio-symbolic process, are always set within a macro-social and institutional context crossed by relations power.

Key words authors

Labor identities, work, subjectivity, Latin America.

Key words plus

Social Psychology, Identity, Chile. 


\section{Antecedentes}

La pregunta por las articulaciones entre el mundo del trabajo, sus transformaciones contemporáneas y la subjetividad de los trabajadores ocupa un lugar destacado en la actual agenda de investigación de la Psicología Social del Trabajo en América Latina. En el marco de la consolidación a nivel mundial de un nuevo modelo de desarrollo capitalista (flexible, global y de liberalismo avanzado), y atendiendo a los específicos procesos de reestructuración productiva y cambio sociocultural ocurridos en las últimas décadas en los países de la región, diversas investigaciones han buscado comprender las emergentes articulaciones entre trabajo y subjetividad que caracterizan la actual fase de modernidad en América Latina (Battistini, 2004; Nardi, 2006; Pulido-Martínez, 2007; Soto, 2008; Sisto, 2009; Schvarstein \& Leopold, 2005; Spink, 2011; Stecher, 2011, 2012). El objetivo de estos estudios ha sido comprender "los modos como los sujetos vivencian y dan sentido a sus experiencias de trabajo, así como la forma en que las relaciones y los contextos de trabajo producen determinados modos de constitución de sujetos" (Tittoni \& Nardi, 2011, p. 375); esto es, particulares modalidades de pensar, sentir y actuar respecto a uno mismo, los otros y el mundo, en un determinado escenario laboral.

Se destacan dos aspectos de este acumulado de investigaciones recientes sobre subjetividad y trabajo. En primer lugar, y en términos empíricos, la singularidad del proceso de modernización capitalista de América Latina (Domingues, 2009), caracterizado por la histórica heterogeneidad estructural de la matriz productiva y sociocultural de los países de la región, al interior de los cuales coexisten, hasta el día de hoy, mundos del trabajo profundamente diferenciados y desiguales -según sectores económicos, tamaño de las empresas, sector formal o informal, patrón productivo (tradicional, fordista, flexible), entre otros (Antunes, 2001; De La Garza, 2000)-, lo cual conlleva tipos de experiencia y condiciones de subjetivación laboral disímiles. Proceso modernizador caracterizado, asimismo, por un fordismo periférico en gran parte del siglo XX (1930-1980) que no supuso la conso- lidación de un Estado de Bienestar, una sociedad salarial ni una masiva industrialización fordista al modo de los países noratlánticos; así como por un proceso de inserción a la nueva economía global y de adopción de los principios del paradigma productivo flexible, que ha ocurrido de modos diferenciales y/o parciales en los distintos segmentos (más o menos modernos) de la economía, y que coexiste con el mantenimiento de patrones tecno-socioproductivos y matrices culturales de tipo fordista o tradicional (De la Garza, 2000).

En segundo lugar, y en términos conceptuales, este conjunto reciente de estudios sobre subjetividad y trabajo en América Latina destaca por problematizar las nociones de sujeto y de identidad, clásicamente utilizadas en las investigaciones sobre el mundo laboral. Se observa, así, un cuestionamiento de las tradiciones estructurales, en sus versiones funcionalistas o marxistas, para las cuales es la posición del individuo en las estructuras socioeconómicas y las categorías ocupacionales lo que determina la particular forma de conciencia y acción (subjetividad) del sujeto en tanto trabajador; así como un cuestionamiento de las tradiciones psicologicistas, para las cuales serían ciertos rasgos intrapsíquicos de la personalidad los que definirían una cierta identidad -estable y hasta cierto punto independiente del contexto laboral- del individuo en tanto trabajador (De la Garza, 2000; Du Gay, 1996). Contra esas tradiciones, predominantes en la sociología y psicología del trabajo de gran parte del siglo XX, muchas de las investigaciones recientes se caracterizan por utilizar conceptualizaciones orientadas a aprehender el carácter procesual, discursivo, tensional, relacional, históricamente situado y contextualmente arraigado de los procesos de configuración de las subjetividades laborales, en los que se anudan y actualizan, de formas heterogéneas y singulares, las relaciones de poder, el carácter constrictivo de las instituciones, los discursos sociales hegemónicos, así como la capacidad de agenciamiento, resistencia y creación de sentido de los sujetos individuales y colectivos (Battistini, 2004; De La Garza, 2006; Stecher, 2011).

Este artículo busca contribuir a este campo de debates e investigación psicosocial sobre trabajo 
y subjetividad en la actual fase de modernización de América Latina, a partir de la presentación de un modelo conceptual orientado al estudio de las subjetividades laborales. Dicho modelo, de raigambre critico-interpretativo, propone como categoría central la noción de "identidad laboral", la que es conceptualizada a partir de una articulación de los desarrollos del interaccionismo simbólico, de la psicología cultural y narrativa y de la tradición de la teoría crítica. A lo largo del artículo, se busca relevar las implicancias del modelo propuesto para la investigación empírica, discutir su pertinencia para el estudio de la realidad latinoamericana, así como destacar su especificidad respecto a otros abordajes teóricos de las subjetividades laborales.

\section{El estudio de las identidades laborales desde una perspectiva crítico-interpretativa}

El concepto de identidad laboral que proponemos se inscribe en una discusión teórica más amplia sobre los procesos de construcción identitaria en el mundo social (Dubar, 1991; Jenkis, 1996; Lawler, 2002). En este primer apartado se propone una definición general del concepto de "identidad”, así como, derivado de esta, una primera aproximación al concepto de "identidad laboral", las cuales se basan en una articulación de los desarrollos del interaccionismo simbólico (Blumer, 1982; Mead, 1972), de la psicología cultural y narrativa (Bruner, 1991, 2003; Crossley, 2003; Ricoeur, 1996), y de ciertos aportes de la tradición crítica de las Ciencias Sociales (Bourdieu \& Wacquant, 1995; Habermas, 1990).

\section{La identidad como producción simbólica y proceso social}

Desde dichas coordenadas teóricas podemos entender la identidad de los sujetos individuales como una construcción simbólica, articulada narrativamente, que se va configurando en el crisol de diversos escenarios de interacción social y a partir de la movilización de diferentes referentes simbólicos presentes en la cultura, y con base en la cual el actor configura -a partir de un juego permanente de relaciones de similitud y diferencia con "otros" y de identificación con diversas identidades colectivas- un cierto sentido de sí mismo, de las demás personas y de su particular posición en el mundo social (Giménez, 1997; Larraín, 2005). Dicho sintéticamente, la identidad es el sentido de sí mismo, inscrito en la forma simbólica de una narrativa (narrativa identitaria), a partir del cual un agente individual interpreta su lugar en el espacio social, orienta su acción en el mundo, se hace distinguible (identificable) -para sí mismo y para los otros- y construye un sentido de continuidad, singularidad y mismidad a lo largo de su biografía personal y a través de distintos dominios de experiencia e interacción (Larraín, 2005; Thompson, 1998). Los procesos de construcción identitaria se dan al interior de (y participan de la reproducción de) campos sociales institucionalmente estructurados y atravesados por relaciones de poder (Thompson, 1993). De este modo, en la configuración de las identidades, se expresa una permanente dialéctica entre, por un lado, las posibilidades de creación de sentido y reflexividad que poseen los actores individuales y, por otro lado, las restricciones institucionales que condicionan estructural y "exteriormente" los distintos campos de interacción social donde se forjan las identidades, así como los recursos y los materiales simbólicos a los cuales tienen o no acceso los distintos actores para interpretar la propia experiencia y construir una particular narrativa sobre sí mismos (Bourdieu \& Wacquant, 1995; Giménez, 1997).

\section{Las identidades laborales}

En el marco de esta formulación teórica general sobre las identidades, y siguiendo de cerca los aportes de Dubar (1991, 1998), se propone conceptualizar a las identidades laborales como producciones simbólicas articuladas narrativamente que expresan las modalidades específicas en que un trabajador entiende y experimenta su espacio laboral, significa a los otros con los que se relacio- 
na en el trabajo y se define a sí mismo como un actor singular al interior de ese espacio social. La identidad laboral es la historia de sí mismo como trabajador que (se) cuenta un agente individual inserto en un mundo del trabajo específico, y en la cual cristaliza una particular interpretación de sí mismo como trabajador, del escenario laboral (empresa, organización, etc.), del proceso de trabajo y de los otros actores que participan de dicho escenario laboral (compañeros, clientes, jefaturas, etc.). Dicho en otros términos, toda identidad laboral da cuenta de una particular modalidad de relación subjetiva con el trabajo, de un específico modo, vivencialmente encarnado y narrativamente articulado, de interpretar y dotar de sentido la experiencia laboral (Dubar, 1998).

La identidad laboral no es, así, una esencia anclada en la persona del trabajador (visión psicologicista) ni un derivado mecánico de la posición que este ocupa en un determinado organigrama o categorización socio-ocupacional (visión estructural-funcionalista). Es una construcción simbólica que le permite al trabajador, en un particular escenario laboral, hacer significativas sus acciones, lograr un cierto sentido de distinción, singularidad y continuidad de la experiencia, al mismo tiempo que construir un sentimiento de pertenencia, semejanza e integración social (Sisto, 2009; Thompson, 1998). La construcción de la identidad laboral se lleva a cabo en el crisol de las interacciones cotidianas dentro del trabajo y de la movilización de los recursos simbólicos (referenciales identitarios) presentes en el escenario laboral (Battistini, 2004). Pero también puede implicar la movilización de significados diversos (e. g., respecto de sí mismo, del trabajo, de la sociedad) que cada trabajador porta, producto de una biografía particular, de una singular historia de experiencias formativas y/o laborales previas, así como de su inserción en otros mundos sociales (familia, amistades, ocio, vida política, comunidades territoriales y/o religiosas, etc.) en los que participa. Aspectos todos estos condicionados por la posición del actor en ciertas categorías socioeconómicas, de género, generación, ocupacionales y étnicas dentro de la estructura social.

\section{Conceptualizando e investigando las identidades laborales desde una perspectiva narrativa, interaccionista y crítica}

En este segundo apartado, se desarrolla en mayor detalle el concepto de identidad arriba propuesto, profundizando en sus distintas dimensiones, explicitando sus fundamentos teóricos y discutiendo sus implicancias para la conceptualización y el estudio de las identidades laborales.

\section{La construcción narrativa de las identidades}

El concepto de identidad laboral propuesto se inscribe en el denominado giro narrativo de las Ciencias Sociales (Lieblich \& Tuval-Mashiach, 1998; Riessman, 2003), y en particular en los desarrollos de la psicología cultural y narrativa (Bruner, 1991; Crossley, 2003). De estas tradiciones se destacan tres aspectos.

En primer lugar, la idea de que el sentido de sí mismo que construye un actor social (su identidad) se configura narrativamente a partir de la elaboración de un relato de su propia historia. Como ha señalado la psicología cultural (Bruner, 1991, 2003), es a través de la elaboración de una narrativa que los sujetos proveen de coherencia y continuidad a su experiencia, que la dotan de sentido, que construyen una imagen de sí mismos, del mundo y de los otros, y que participan de la acción conjunta y la negociación de significados dentro de su cultura. El sí mismo reflexivo construye los sentidos con los que orienta su acción en el mundo a partir de la elaboración permanente de narrativas en que el individuo queda ubicado como el actor de una historia determinada por el pasado pero abierta al cambio y al porvenir. Como señala Bruner (2003)

He afirmado que creamos y recreamos la identidad mediante la narrativa, que el yo es un producto de 
nuestros relatos y no una cierta esencia por descubrir cavando en los confines de la subjetividad. Y a esta altura está demostrado que sin la capacidad de contar historias sobre nosotros mismos no existiría una cosa como la identidad. (p. 122)

Las narraciones que elaboran los actores sociales, para sí mismos y para los otros, más que como vías de acceso o expresiones de una identidad intrapsíquica estable y preconstituida, o como descripciones fidedignas de los acontecimientos y el mundo exterior, deben ser entendidas como recursos interpretativos a partir de los cuales los agentes individuales configuran un sentido de sí mismos, de los otros y del mundo social en que habitan. Como afirma Lawler (2002), todos los sujetos (se) cuentan historias sobre sus propias vidas, y es a través de dichas historias que logran dotar de sentido e interpretar sus experiencias, sus relaciones con los otros y su lugar en el mundo.

En segundo lugar, la tesis de que las narrativas identitarias se configuran a partir de la movilización de diversos referentes simbólicos presentes en el particular contexto sociocultural donde están insertos los sujetos, y suponen siempre una orientación hacia los otros. Es decir, si bien cada narrativa identitaria es una producción simbólica del agente individual, esta está necesariamente elaborada a partir de las narrativas públicas y las categorías socio-discursivas presentes en la cultura, y ha sido forjada en el contexto de diversas interacciones sociales y tramas relacionales donde los "otros" juegan un rol constitutivo. Como señalan Gergen (1997) y Gubrium y Holstein (1998), producir una narrativa identitaria coherente, legítima y significativa para mí mismo y los otros, y que me permita ser reconocido e incluido dentro del espacio social, exige atender a las reglas de narración, a las historias canónicas dentro de la sociedad, a los valores culturales, así como a los modelos de identidad presentes y legitimados dentro de la cultura (general o particular) a la que pertenezco (Somers, 1994). En tanto somos agentes culturales inscritos en un orden socio-simbólico que nos antecede, y en tanto nos narramos considerando las expectativas de los otros y buscando que nos reconozcan, las matrices culturales, los imaginarios y discursos hegemónicos y los ideales de sujeto legitimados socialmente son siempre un elemento constitutivo de toda narrativa identitaria.

En tercer lugar, una cierta conceptualización de lo que es una narrativa, del modo en que se compone y en el que debe ser analizada. Una narrativa es un particular tipo de discurso organizado como un relato, que contiene una línea de transformación a través del tiempo (pasado, presente, futuro) y donde están presentes personajes, situaciones y acciones diversas ligadas y articuladas coherentemente en una trama narrativa (Lawler, 2002; Riessman, 1993). La trama narrativa es el elemento central del relato en tanto articula bajo una lógica y orientación global un conjunto de eventos heterogéneos que aparecen causal y/o secuencialmente conectados entre sí como episodios de una única historia. El significado de cada uno de esos episodios solo puede ser reconstruido bajo el marco de la trama global en la que está inserto, esto es, a la luz de la constelación de relaciones temporales y espaciales que reúne y articula en un mismo y único relato a los distintos personajes, acciones y eventos de la narrativa.

Para el caso de las narrativas identitarias -los relatos que en primera persona construyen los actores sociales sobre su propia vida o parte de ella- es central relevar la dimensión temporal y evaluativa. Respecto a lo primero, se plantea que toda narrativa identitaria implica una estructura temporal, donde todo lo que soy en el presente se entiende como lo que he llegado a ser a la luz de una cierta historia pasada y como lo que me habilita o restringe, de cara al futuro, a ciertas posibilidades. Como señala Crossley (2003), al interrogar a alguien por una experiencia o por su vida, lo que uno obtiene necesariamente es una narrativa con una estructura temporal pasadopresente-futuro, esto pues toda experiencia y toda vida requiere esa inscripción temporal para adquirir sentido. Así, el sentido de sí mismo (la identidad) en un momento dado de una persona es una significación que emerge en el crisol de una historia que señala aquello que ha sucedido y que anticipa aquello que puede llegar a suceder. Respecto a lo segundo (la dimensión 
evaluativa), se plantea que todo sujeto al momento de contar su historia establece, explícita o implícitamente, un juicio de valor respecto a aquellos acontecimientos que relata. Así, al configurar y relatar su narrativa identitaria, todo agente, por un lado, cuenta una historia -un conjunto de hechos acaecidos- y, al mismo tiempo, se posiciona evaluativamente ante aquello que cuenta a partir de un conjunto de juicios y valoraciones morales que clasifican como buenos/malos, correctos/incorrectos, adecuados/inadecuados los distintos acontecimientos, personajes y acciones presentes en la historia. Es en esa articulación entre la historia que se cuenta (told) y el modo valorativo en que se cuenta (telling) que se va configurando la identidad del actor social o narrador (teller) (Hiles, Cermack \& Chrz, 2009).

\section{Estudiando las identidades laborales como narrativas identitarias}

Estas tres puntualizaciones permiten fundamentar teóricamente la conceptualización de las identidades laborales como narrativas identitarias, al mismo tiempo que arrojan luces sobre cómo investigar las mismas. Estudiar las identidades laborales de un colectivo de trabajadores supone invitarlos a elaborar una narrativa sobre su actual experiencia de trabajo (cómo llegó a su actual trabajo, cómo es este actualmente, cómo se visualiza hacia el futuro), visibilizando los referentes simbólicos intra y extralaborales (ideales de sujeto, imaginarios, discursos sociales, narrativas canónicas) a partir de los cuales esta se compone y develando, a partir del análisis global (no segmentado) de la historia que se cuenta (su contenido y su estructura formal) y del modo (juicios valorativos) en que se cuenta, el particular sentido de sí mismo en tanto trabajador (identidad laboral) que emerge del relato (Lieblich \& Tuval-Mashiach, 1988). El análisis, a partir de un ejercicio inductivo de comparación constante de las narrativas o casos individuales, puede arrojar la existencia de un único tipo o perfil de identidad laboral en el colectivo de trabajadores estudiado, o puede revelar la presencia de diferentes tipos de identidad laboral, entendidos estos como modali- dades prototípicas de narrar e interpretar la experiencia laboral en ese escenario de trabajo (Dubar, 2001; Stecher, 2012).

\section{La identidad como proceso social en el marco de la interacción simbólica}

Además de como narrativa identitaria, la identidad laboral debe ser comprendida como un proceso social de interacciones mediadas simbólicamente al interior de un particular escenario laboral. Así, el concepto propuesto se inscribe en la tradición teórica del interaccionismo simbólico (Blumer, 1982; Dubar, 1991; Mead, 1972), donde la identidad -el significado de sí mismo que construyen los individuos- se entiende no como algo dado, sino como un emergente de las interacciones sociales mediadas por el lenguaje en las que participan los actores sociales. De esta tradición teórica se destacan tres aspectos que están en la base del concepto de identidad laboral que se propone.

En primer lugar, la reflexividad del sí mismo y el carácter procesual de las identidades. Respecto a la reflexividad, se plantea que el agente humano es un sí mismo (self) que tiene la capacidad de tomarse como objeto, de interrogarse, de interpretar y reformular el sentido de sí mismo y del mundo, en el curso de la acción cotidiana y de las prácticas sociales en las que participa. Con base en esa capacidad reflexiva las personas llevan a cabo continuamente un "trabajo identitario" (identity work), un esfuerzo activo (muchas veces no consciente) por configurar un sentido de sí mismos (identidad) que oriente sus acciones y los provea de un sentimiento de coherencia, singularidad, continuidad y pertenencia en el espacio social (Alvesson, 2010; Giddens, 1997). Estudiar las identidades exige, así, reconocer esa dimensión reflexiva e interpretativa de la agencia humana y atender, no solo a la identidad en tanto sentido de sí mismo ya estabilizado, sino al trabajo identitario continuo y permanente -el que se incrementa en coyunturas de incertidumbre, crisis y cambio- que está en la base de los procesos de construcción identitaria. Respecto al carácter procesual de las identidades, se postula la necesidad de estudiar las identidades como un "siendo" más que 
como un "es", como una producción simbólica que se configura, recrea y está abierta al cambio en el flujo de las interacciones en las que participan y en las que quedan situados los actores sociales (Bruner, 1991). Estudiar la identidad exige, desde esta perspectiva, tomar distancia de las tradiciones que tienden a reificarla, cosificarla y fijarla como una entidad psicológica o sociológica estable y continua, relevando, por el contrario, el carácter procesual que la define en tanto emergente de las interacciones sociales, del trabajo identitario del individuo y de la apropiación de las formas simbólicas presentes en la cultura (Jenkins, 1996).

En segundo lugar, la centralidad de las dinámicas de hetero-categorización y auto-categorización en los procesos de construcción de identidad, así como las tensiones identitarias que caracterizan a los mismos. Como señala Mead (1972), el sentido de sí mismo de una persona está siempre mediatizado por las expectativas de los otros significativos y de los distintos colectivos de la sociedad (otro generalizado), siendo así que el modo en que un actor se define e interpreta a sí mismo (su identidad) es en gran medida el resultado de la internalización que ha hecho del modo como los otros lo reconocen, lo categorizan y definen en el espacio social (Larraín, 2005). Estudiar los procesos identitarios exige atender a la diversidad de categorías socio-discursivas o papeles sociales, institucionalmente formalizados o cotidianos e informales, en que los individuos son posicionados por otros (instituciones, personas, colectivos, medios de comunicación, etc.), a lo largo de su vida y en distintos contextos de interacción social (Jenkins, 1996). Ahora bien, lo anterior no supone una conceptualización del Sí mismo y la identidad en términos de mera pasividad, conformismo y reproducción mecánica del orden sociosimbólico (Larraín, 2005). Siguiendo la distinción de Mead entre el "mí" -la dimensión objetivada, reproductora y convencional- y el "yo" -la dimensión dinámica, creativa y contingente- como dos fases o aspectos del Sí mismo, es posible plantear que en la vida social las personas no se limitan a adoptar mecánicamente las actitudes, representaciones, interpelaciones simbólicas y categorizaciones que reciben de los otros y la sociedad, sino que llevan a cabo un activo trabajo de apropiación, re-interpretación e incluso resistencia respecto a esas mismas categorizaciones que los otros depositan sobre ellos. Atender a esta dimensión creativa y agencial del sí mismo implica reconocer que los individuos no son solo objeto de (hetero)categorizaciones y atribuciones externas de sentido, sino que también llevan a cabo activamente procesos de auto-categorización basados en la identificación con distintos grupos, personas e instituciones, y en relación a los cuales construyen fuertes sentidos de pertenencia (Solis, 2009).

Siguiendo esta misma matriz conceptual Dubar $(1991,1998)$ plantea que estudiar los procesos identitarios, en un momento y en un contexto dado, exige prestar atención simultáneamente, tanto a las categorizaciones sociales y a las atribuciones de identidad que recaen sobre el individuo en el marco de sus interacciones sociales (lo que denomina como "identidad para otro") como al modo en que dicho individuo se apropia, resignifica e incluso resiste dichas (hetero)categorizaciones a la luz de el sentido de sí mismo (lo que denomina como "identidad para sî"), que ha ido moldeando a lo largo de su biografía (lo que he sido, lo que soy y lo que quiero llegar a ser) y con el cual se inserta en y empieza a participar de un cierto contexto de interacción.

Esta distinción entre procesos de heterocategorización (identidad para otro) y autocategorización (identidad para sí) permite, a su vez, visibilizar la presencia de diversas tensiones identitarias en los procesos de construcción de identidad. Tensiones que emergen de las brechas que pueden existir entre las modalidades de auto-reconocimiento (identidad para sí) y hetero-reconocimiento (identidad para otro) en un momento y espacio social determinado, pero que también pueden emerger al interior de la misma identidad para sí -por ejemplo, una brecha y diferencia entre la identidad heredada del actor social desde su linaje familiar y sociocultural, la identidad para sí asumida en el presente y/o la identidad anhelada hacia el futuro- o de la misma identidad para otro - producto, por ejemplo, de reconocimientos diversos y diferentes desde 
distintos actores o campos de interacción social, o de interpelaciones y categorizaciones disímiles recibidas simultáneamente desde un mismo otro significativo- (Bajoit, 2003).

En tercer lugar, se destaca la importancia de las dinámicas de construcción de semejanzas y diferencias, así como la centralidad de las identidades colectivas, en los procesos identitarios. Respecto a lo primero, hay que señalar que el sentido de distinguibilidad para uno mismo y los otros en el espacio social que provee la identidad, se asienta en gran medida en el establecimiento de relaciones de semejanza (identificación) y diferenciación con otras personas, colectivos o categorías, lo que expresa el carácter relacional de toda identidad (Hall, 2003). Una noción útil para pensar esta dinámica de diferenciación y semejanza es la de referenciales identitarios propuesta por Battistini (2004), la que da cuenta del conjunto de anclajes o marcas que nos sirven como puntos de referencia desde los cuales decir(nos) quienes somos. Pueden ser objetos, personas, acciones, colectivos, categorías, instituciones, etc. a partir de los cuales - marcando nuestra cercanía (semejanza) o nuestra distancia (diferenciación) a ellos- configuramos una particular autointerpretación de nosotros mismos. Es importante destacar, a su vez, que estas dinámicas de semejanza y diferencia presentes en todo proceso identitario van ligadas a actos de valoración y juicio moral, que implican, por lo general, una valoración más positiva de aquellas personas o categorías de las que me siento semejante y un juicio más negativo de aquellos "otros" respecto a los cuales, por oposición y/o diferenciación, me defino a mí mismo (Giménez, 1997; Thompson, 1993).

Respecto a lo segundo, y muy ligado a las dinámicas de identificación y semejanza, se destaca la centralidad de las identidades colectivas y de los sentidos de pertenencia en los procesos de construcción de las identidades. La identidad de todo agente individual se define en gran medida en función de la pluralidad de sus pertenencias a distintos colectivos sociales presentes en sus particulares marcos socioculturales y espacios de interacción (Giménez, 1997; Larraín, 2005). Es decir, en la construcción del sentido de sí mismo los individuos siempre definen lo que son a partir de reconocer su pertenencia a ciertos colectivos con los que activamente se identifican. Responder a la pregunta de quién soy, de quién quiero ser, de cómo quiero que me reconozcan, implica necesariamente la referencia a un conjunto de colectivos o "comunidades imaginadas" - como la profesión, la religión, la empresa, el sindicato, el partido político, la nación, el barrio, la etnia, el género, etc.- a los que el sujeto se siente subjetivamente vinculado y que operan como espacios de pertenencia, matrices simbólicas y fuentes de sentido.

\section{Estudiando las identidades laborales como procesos de interacción socio-simbólica}

Estas tres puntualizaciones permiten fundamentar teóricamente la concepción propuesta de la identidad laboral como un proceso social de construcción de significado anclado en las prácticas, simbólicamente mediadas, de interacción con "otros" (jefaturas, clientes, colegas, sindicatos, instituciones, colectivos, etc.) en el espacio del trabajo, pero también fuera del espacio laboral. Estudiar las identidades laborales exige analizar el modo en que los sujetos, al interior de un contexto laboral específico, pero también producto de las formas de reconocimiento que reciben en otras esferas sociales, van configurando un cierto sentido de sí mismos en tanto trabajadores. Esto a partir de un activo trabajo identitario que supone el establecimiento permanente de relaciones de semejanza y diferencia con otros actores/grupos/ categorías socio-laborales, así como la construcción de diversos sentidos de pertenencia e identificación respecto a distintas identidades colectivas (colectivos profesionales, sindicatos, categorías ocupacionales, oficios, etc.). Más que como un núcleo estable y fijo, las identidades laborales deben ser interrogadas reconociendo su carácter procesual, estando abiertas al cambio en función de procesos de transformación en los contextos laborales (una huelga, una fusión de la empresa, un ascenso, un cambio de tipo de contrato, etc.), o en otros dominios de la vida social del actor (por ejemplo el nacimiento de un primer 
hijo en mujeres trabajadoras jóvenes). Estudiar las identidades laborales exige estudiar, no solo el producto o significado de sí mismo ya estabilizado en una narrativa, sino también el proceso mismo de construcción identitaria al interior de ciertos contextos específicos de trabajo, y por parte de actores que portan las marcas simbólicas de sus singulares trayectos biográficos y que participan cotidianamente de otros mundos sociales no laborales ${ }^{1}$. Se trata de analizar las complejas dinámicas de heterocategorización y auto-categorización, las tensiones identitarias que estas conllevan y el modo en que en el crisol de las mismas el trabajador configura un sentido de sí mismo a partir de la elaboración de una particular narrativa de su experiencia e historia laboral. En los términos de Dubar (1991, 2001), se trata de analizar las identidades laborales en tanto producciones simbólicas que emergen en el espacio de la interacción social a partir de la articulación de la identidad para sí y la identidad para otros. Esto supone por parte del investigador una aproximación tanto biográfica y diacrónica -orientada a reconstruir la biografía y trayectoria (pre)laboral (modelos familiares de trabajo, modelos de trabajo según género, experiencias educativas e identificación con ideales socioprofesionales, primeras inserciones al mercado laboral, etc.) y los sentidos de sí mismo que en tanto trabajador el sujeto ha ido construyendo a lo largo de esa historia (identidad para sí) - como relacional y sincrónica -orientada a dar cuenta de las distintas objetivaciones, categorizaciones, reconocimientos y adscripciones identitarias que el sujeto recibe desde los otros (jefaturas, colegas, clientes, sindicato) en el marco de su particular inserción en el presente en un proceso de trabajo y una empresa/organización específica (identidad para otros).

1 El estudio de las identidades laborales requiere analizar, junto al contexto laboral específico en que el trabajador está inserto en el presente, los otros mundos sociales en que participa dicho sujeto en ese mismo presente, así como su particular trayecto biográfico (origen familiar, formación, experiencias laborales previas, etc.). Las identidades laborales se forjan en el marco de un complejo trabajo identitario en que el agente individual moviliza tanto los patrones de significado de su actual espacio de trabajo, como otros referentes de sentido -respecto a lo que es ser trabajador- provenientes de sus otros mundos presentes de experiencia (familia, ocio, amistades) y de su trayecto biográfico (laboral y extralaboral) pasado (Dubar, 2001; Wilkis \& Battistini, 2005).

\section{El carácter institucionalmente situado y políticamente disputado de los procesos identitarios}

La conceptualización de identidad laboral propuesta, además de nutrirse de las corrientes narrativas e interaccionistas, incorpora aportes de las denominadas tradiciones críticas de las Ciencias Sociales, especialmente de la teoría crítica de la Escuela de Frankfurt (Habermas, 1990; Leiva, 2005). Destacamos tres aspectos de estas tradiciones.

En primer lugar, la importancia de transitar desde una concepción meramente simbólica de la cultura (y de los procesos identitarios que en su crisol se forjan), a una concepción estructural de la cultura. Esta, si bien reconoce la importancia y centralidad de las formas simbólicas en la vida social, destaca el hecho de que dichas formas simbólicas y los significados que vehiculizan están siempre insertos en contextos sociales estructurados (Thompson, 1993). Esto implica que las narrativas identitarias que construyen los actores sociales, así como los materiales simbólicos que movilizan para la construcción de las mismas, deben ser analizados reconociendo el contexto histórico, el marco institucional y la estructura social al interior del cual se insertan. El análisis de las formas simbólicas -entre ellas la identidadal interior de un particular contexto o mundo social, exige una reconstrucción de los aspectos socioinstitucionales o socioestructurales específicos que enmarcan y condicionan fuertemente las dinámicas locales de interacción y los recursos semiótico/discursivos que constituyen la materia prima de los procesos de construcción de identidad. De este modo, se busca evitar el riesgo de un "culturalismo autorreferencial" que no atiende a las dimensiones estructurales e institucionales que enmarcan la vida social, y que suponen una distribución desigual de recursos y capitales entre los distintos actores según aspectos como la clase, el género, la etnia, la edad, y que condicionan por tanto fuertemente las producciones simbólicas y las lógicas de acción y construcción de sentido de los actores sociales (Bourdieu \& Wacquant, 1995; Thompson, 1993). 
En segundo lugar, la necesidad de atender a las articulaciones entre las formas simbólicas y las relaciones de poder y dominación presentes en la sociedad. Es decir, la cultura y las identidades no son solo textos a ser leídos e interpretados, sino campos de disputas y luchas sociales donde distintos actores, con intereses muchas veces antagónicos, buscan imponer y volver hegemónicas ciertas formas de categorización e interpretación del mundo en función de sus particulares intereses y proyectos de sociedad (Fairclough, 1992). El estudio de las formas simbólicas, entre ellas las identidades, exige atender al modo en que las mismas pueden contribuir -a través de procesos de legitimación y naturalización del orden social- a sostener relaciones de dominación y legitimar el poder de los grupos dominantes, así como al modo en que en las sociedades modernas los distintos actores sociales cuentan con recursos muy desiguales para difundir particulares perspectivas y significados (Thompson, 1993). Asimismo, es importante atender, no únicamente a la hegemonía y dominación simbólica de las élites socioeconómicas, sino también al modo en que los diferentes actores subalternos luchan por resistir, reinterpretar y/o deconstruir determinados patrones de significación y modelos identitarios, logrando instalar muchas veces - bajo ciertas condiciones y en el marco de luchas por el reconocimiento- contra-discursos y modelos identitarios alternativos a los hegemónicos (Bourdieu \& Wacquant, 1995; Fairclough, 1992).

En tercer lugar, la importancia de estudiar los procesos de construcción de identidad desde una perspectiva crítica que contribuya a la democratización y emancipación de la sociedad. Se trata, así, en la tradición de la teoría crítica (Leiva, 2005), de generar un saber que más que limitarse a constatar lo dado y reificar el orden socioinstitucional y/o sociosimbólico existente, sea capaz de visibilizar las (legitimadas) injusticias, las (opacadas) contradicciones y las (naturalizadas) desigualdades del presente, abriendo así nuevas posibilidades de autocomprensión y acción social para los sujetos sociales (Habermas, 1990). Este esfuerzo requiere un trabajo permanente de análisis y deconstrucción de los mecanismos de producción y reproducción social -donde la cultura y las identidades juegan un rol central-contrastando la realidad "positivizada" del orden social instituido, con un cierto horizonte normativo (autonomía, igualdad, democracia) que, por contraposición, ilumina las injusticias del presente y permite avizorar, desde el análisis (y dentro) de esa misma realidad sociohistórica, otras formas alternativas y más justas de ser (Leiva, 2005).

\section{Estudiando las identidades laborales en sus contextos socio-estructurales}

Estas tres puntualizaciones permiten precisar y fundamentar el talante crítico del concepto de identidad laboral propuesto, al mismo tiempo que arrojan luces adicionales sobre cómo investigar las mismas. Estudiar las identidades laborales exige hacer un recorte analítico de la sociedad, poniendo el foco en un particular escenario de trabajo en el que están insertos un conjunto de actores sociales. Dicho escenario de trabajo debe ser detalladamente reconstruido, no solo en lo que respecta a sus aspectos socio-simbólicos (culturas del trabajo o patrones de significación de la experiencia laboral producidos por los distintos actores) y a sus lógicas cotidianas de interacción social, sino también en lo que respecta a sus aspectos socio-institucionales y socio-estructurales que enmarcan y condicionan los recursos simbólicos y el campo de interacciones en el crisol del cual los trabajadores configuran sus identidades laborales. En términos más específicos, el estudio de las identidades laborales en un particular escenario de trabajo exige dar cuenta de aspectos tales como el proceso de trabajo, las formas de empleo, las relaciones laborales capital-trabajo, el patrón tecnológico, el perfil sociodemográfico de la fuerza de trabajo, las reglas, jerarquías, relaciones de poder y modelo productivo de la empresa u organización, así como las instituciones externas en las que esta está situada: mercado laboral, sector productivo, legislación laboral, redes nacionales y globales de producción y consumo, etc. (Thompson, 1993). Estudiar las identidades laborales, exige así, reconstruir un particular escenario de trabajo entendido como un mundo social en que se articulan elementos socio-institucionales y socio-simbólicos, 
los cuales enmarcan el despliegue cotidiano de un conjunto de interacciones sociales, $\mathrm{y}$ al interior del cual los trabajadores construyen sus narrativas identitarias $^{2}$. La tarea del investigador es, justamente, ofrecer un modelo interpretativo de las narrativas identitarias que, además de iluminar los sentidos de sí mismo que movilizan y los recursos simbólicos con que se componen, y de dar cuenta de los procesos de interacción en que se forjan, permita comprender la incidencia de los aspectos socioestructurales en la producción de las mismas, así como el modo en que dichas formas identitarias contribuyen a la reproducción, o eventualmente tensionamiento y transformación, de ese particular escenario laboral.

Por otro lado, el análisis de las identidades laborales -especialmente en las grandes empresas u organizaciones- exige prestar atención al modo como las gerencias buscan imponer ciertos modelos de identidad laboral, de modo de poder regular, controlar y orientar la subjetividad de los trabajadores en función de ciertos objetivos estratégicos de la organización y/o sus propietarios (Alvesson \& Willmott, 2002; Stecher, 2010). Analizar los procesos identitarios exige, así, atender al modo en que las particulares categorías, modelos de identidad, discursos e interacciones simbólicas presentes en un determinado espacio laboral, son parte y participan de específicas lógicas de control y regulación (racionalidades de gobierno) funcionales a los intereses de ciertos actores específicos dentro de la unidad productiva y/o a las dinámicas más globales presentes en el nuevo modelo de desarrollo capitalista. Al respecto, es fundamental atender en el contexto contemporáneo a la circulación de los discursos que caracterizan a la "cultura" o al "espíritu" del nuevo capitalismo, con su nuevo ideal de trabajador flexible, emprendedor, polivalente, individualizado,

2 Desde esta perspectiva no resulta posible estudiar la identidad laboral de un sujeto en abstracto, sin considerar su particular contexto de trabajo. Al respecto, este es uno de los problemas de los argumentos de autores como Sennett $(2002,2006)$ quienes muchas veces hablan de una nueva identidad o subjetividad laboral en el capitalismo global y flexible en términos sumamente generales y sin referencia a contextos laborales específicos, o extrapolando la situación de un único contexto laboral (habitualmente grandes y modernas corporaciones multinacionales) a la totalidad de los mundos del trabajo. colaborador, autorregulado y leal a su organización (Boltanski \& Chiapello, 2002; Sennett, 2006). Esto, tanto en la sociedad en su conjunto, como en el nivel más local de las empresas y sus diversas prácticas de gestión y organización flexible.

Asimismo, como hemos señalado, el análisis de las identidades laborales exige dar cuenta no únicamente de las estrategias de la racionalidad dominante; es importante atender también, como señala De Certau (1996), a las tácticas locales y furtivas de resistencia de los agentes, los que construyen sentidos diversos y apropiaciones divergentes a las esperadas por la racionalidad de gobierno, abriendo en el ámbito local de lo cotidiano espacios de creación de sentido no subordinados al orden y racionalidad dominante, y que incluso eventualmente podrían llegar bajo ciertas condiciones de organización colectiva a debilitar o subvertir el mismo (Abal, 2007).

Por último, hay que señalar la importancia de producir un tipo de investigación social que, a través de su capacidad de problematización e interrogación crítica de los mundos del trabajo y de su articulación con diversos actores laborales, políticos o de la sociedad civil, contribuya a la democratización de la sociedad y a una disminución de la asimetría que caracteriza la relación capital-trabajo en el mundo contemporáneo.

\section{Reflexiones finales}

Se ha presentado un modelo teórico psicosocial para conceptualizar y estudiar las identidades laborales basado en los aportes de la tradiciones narrativa, interaccionista y crítica de investigación social. Dicho modelo busca ser un aporte al desarrollo de la Psicología Social del Trabajo en América Latina, específicamente en lo que respecta a la investigación sobre las emergentes articulaciones entre trabajo y subjetividad en la actual fase de modernización de los países de la región. Para finalizar, se destacan dos aspectos del modelo conceptual propuesto que dan cuenta, por un lado, de su potencial empírico para el análisis de los heterogéneos, desiguales y transformados mundos del trabajo en América Latina, así como, por otro lado, de su potencial teórico en 
tanto aporte al esfuerzo de repensar el concepto de identidad (laboral) como clave de teorización y análisis de los procesos psicosociales en el mundo contemporáneo.

Respecto a lo primero, es importante destacar que el modelo propuesto puede ser utilizado en el estudio de diferentes escenarios laborales y colectivos de trabajadores, lo que es fundamental considerando la histórica heterogeneidad de la matriz cultural y estructural de América Latina, donde coexisten hoy en día lógicas productivas tradicionales, fordistas y posfordistas, y donde el sector informal de la economía tiene un peso significativo en el mercado laboral. El carácter amplio y psicosocial de las categorías básicas del modelo -narrativas, espacios de interacción social y modos de hetero y autocategorización, marcos socioinstitucionalespermite su aplicación en mundos socio-laborales diversos. Por otro lado, al centrarse en el nivel de análisis del agente individual como actor social, y al reconocer la centralidad para la comprensión de las identidades laborales de los trayectos biográficos previos (laborales y no laborales) de los sujetos (identidad para sí), y de los otros mundos sociales en los que participa en el presente (familia, redes sociales, amistades, ocio, organizaciones, etc.), el modelo conceptual evita el riesgo de sobreestimar el peso de las lógicas, categorizaciones e imaginarios organizacionales, empresariales y productivos en las identidades laborales. Estos, si bien son centrales, parecen tener muchas veces un peso relativo menor en el contexto de sociedades como las de América Latina, donde nunca se consolidó del mismo modo que en los países noratlánticos una modernización industrial fordista (y posfordista) ni un imaginario laboral y un ética del trabajo fordista (y posfordista), y donde otros referentes culturales (religiosos, urbano-populares, comunitarios, territoriales) juegan muchas veces un papel importante en las autocomprensiones de sí mismos en tanto trabajadores que construyen los actores sociales (Martucelli, 2010; Spink, 2011). Por último, el modelo conceptual propuesto disminuye el riesgo -en el análisis de las contemporáneas articulaciones entre trabajo y subjetividad- de sobredimensionar los procesos de flexibilización y de expansión de la cultura del nuevo capitalismo y su ideal de trabajador flexible. El modelo conceptual no tiene como objetivo fundamental pesquisar o determinar el grado de flexibilización de un escenario laboral determinado, ni precisar cuán presentes están (o no) los denominados modos flexibles de subjetivación laboral. Su pretensión es analizar los procesos de construcción de las identidades laborales de ciertos actores (que portan una particular identidad para sí, producto de su historia) insertos en el presente en un particular contexto de trabajo, lo que puede suponer o no (algo que se debe determinar empíricamente caso a caso), la presencia de referentes simbólicos del nuevo capitalismo y de lógicas de gestión y organización propias del paradigma productivo flexible.

Respecto a lo segundo, es posible plantear que el modelo conceptual propuesto contribuye a la necesaria tarea de discutir y repensar el concepto de identidad dentro de la psicología social. En esa línea, se ha señalado la importancia de entender la identidad, simultáneamente, como una producción simbólica (narrativa identitaria) y como un proceso micro-social (campo de interacciones sociales espacio-temporalmente situadas, simbólicamente mediadas y culturalmente enraizadas), inserto en el contexto macro-social de escenarios socio-históricos y marcos institucionales "objetivamente" estructurados (Thompson, 1993, 1998). Esta perspectiva releva la centralidad de la noción de identidad en tanto espacio de intensidad teórica que permite interrogar y mediar, teórica y empíricamente, la dialéctica individuo-sociedad y estructura-agencia (Habermas, 1987). Esta concepción de identidad se ubica, en el campo de los debates contemporáneos de la psicología social, en un lugar intermedio entre, por un lado, las posiciones sociocognitivas basadas en la noción de auto-concepto y, por otro lado, las posiciones discursivas y/o postestructuralistas que entienden la identidad como un flujo de posiciones, o efectos contingentes y fragmentarios, en el discurso o en la performatividad de la acción situada (Gergen, 1997; Smith \& Sparkes, 2008). Siguiendo los planteamientos de Ricoeur (1996) y Bruner (1991) se ha propuesto comprender las identidades de los agentes individuales -y por derivación sus identidades laborales- desde abordajes que escapen a la dicotomía reduccionista 
que obliga a entenderlas, o como esencias psicológicas, individuales, autocontenidas e inmutables, o como meros efectos o ilusiones lingüísticas fragmentarias, contingentes y contradictorias (Ezzy, 1998; Larraín, 2005). De este modo, se espera haber contribuido a la tarea de avanzar en una conceptualización psicosocial crítico-interpretativa de las identidades, que dé cuenta del carácter procesual, relacional, narrativo y simbólicamente mediado de los procesos identitarios, que reconozca tanto el rol constrictivo de las estructuras institucionales y discursivas, como la capacidad interpretativa y reflexiva del agente humano, y que permita contribuir, a partir de la visibilización de las articulaciones entre las formas simbólicas y las relaciones de poder, a la democratización de nuestras sociedades y sus espacios laborales.

\section{Referencias}

Abal, P. (2007). El destierro de la alteridad. El caso Wal Mart Argentina. Revista Mexicana de Sociología, 69(4), 688-727.

Alvesson, M. (2010). Self-doubters, strugglers, storytellers, surfers and others: Images of self-identities in organizations studies. Human Relations, 63(2), 193-217.

Alvesson, M. \& Willmott, H. (2002). Identity regulation as organizational control: Producing the appropriate individual. Journal of Management Studies, 39(5), 619-644. doi:10.1111/1467-6486.00305

Antunes, R. (2001). iAdiós al trabajo? Ensayo sobre la metamorfosis y la centralidad del mundo del trabajo. Brasil: Cortez Editora.

Bajoit, G. (2003). Todo Cambia. Análisis sociológico del cambio social y cultural en las sociedades contemporáneas. Santiago de Chile: LOM Ediciones.

Battistini, O. (Comp.). (2004). El trabajo frente al espejo: continuidades y rupturas en los procesos de construcción identitaria de los trabajadores. Buenos Aires: Prometeo.

Blumer, H. (1982). El interaccionismo simbólico: perspectiva y método. Barcelona: Hora.

Boltanski, L. \& Chiapello, E. (2002). El nuevo espíritu del capitalismo. Madrid: Akal.

Bourdieu, P. \& Wacquant, L. (1995). Respuestas por una antropología reflexiva. México: Editorial Grijalbo.
Bruner, J. (1991). Actos de significado. Madrid: Alianza.

Bruner, J. (2003). La fábrica de historias. Buenos Aires: F.C.E.

Crossley, M. L. (2003). Formulating narrative psychology: The limitations of contemporary social constructionism. Narrative Inquiry, 13(2), 287-300.

De Certau, M. (1996). La invención de lo cotidiano. México: Universidad Iberoamericana.

De la Garza, E. (Coord.). (2000). Tratado latinoamericano de sociología del trabajo. México: F.C.E.

De la Garza, E. (Coord.). (2006). Teorías sociales y estudios del trabajo: nuevos enfoques. México: Anthropos.

Domingues, J. M. (2009). La modernidad contemporánea en América Latina. Buenos Aires: Siglo XXI.

Dubar, C. (1991). La socialisation, construction des identités sociales et professionnelles. París: Armand Colin.

Dubar, C. (1998). Trajetórias sociais e formas identitárias: alguns esclarecimentos conceituais e metodológicos. Educação E̊ Sociedade, 19(62), 13-30. doi:10.1590/S0101-73301998000100002

Dubar, C. (2001). El trabajo y las identidades profesionales y personales. Revista Latinoamericana de Estudios del Trabajo, 7(13), 5-16.

Du Gay, P. (1996). Consumption and identity at work. London: Sage.

Ezzy, D. (1998). Theorising narrative identity: Symbolic interactionism and hermeneutics. Sociological Quarterlyl, 39(2), 239-252.

Fairclough, N. (1992). Discourse and social change. Cambridge: Polity Press.

Gergen, K. (1997). Construccionismo social. Aportes para el debate y la práctica. Bogotá: Uniandes.

Giddens, A. (1997). Modernidad e identidad del yo. Barcelona: Península.

Giménez, G. (1997). Materiales para una teoría de las identidades sociales. Instituto de Investigaciones Sociales de la UNAM (documento inédito).

Gubrium, J. \& Holstein, J. (1998). Narrative practice and the coherence of personal stories. Sociological Quarterly, 39(1), 163-187.

Habermas, J. (1987). Teoría de la acción comunicativa. Crítica de la razón funcionalista (Vol. 2). Madrid: Taurus.

Habermas, J. (1990). Conocimiento e interés. Madrid: Taurus. 
Hall, S. (2003). Introducción: ¿Quién necesita "Identidad"? En S. Hall \& P. Du Gay (Coords.), Cuestiones de identidad cultural (pp. 13-39). Buenos Aires: Amorrortu.

Hiles, D., Cermak, I. \& Chrz, V. (2009). Narrative oriented inquiry: A dynamic framework for good practice. En D. Robinson, P. Fisher, T. Yeadon-Lee, S. J. Robinson \& P. M. Woodcock (Eds.), Narrative, memory, and identities (pp. 53-65). Huddersfield, Reino Unido: University of Huddersfield.

Jenkins, R. (1996). Social identity. Londres: Routledge.

Larraín, J. (2005). iAmérica Latina moderna? Santiago de Chile: LOM Ediciones.

Lawler, S. (2002). Narrative in social research. En T. May (Ed.), Qualitative research in action (pp. 242-258). Londres: Sage.

Leiva, G. (2005). La teoría crítica y las tareas actuales de la crítica. Barcelona: Anthropos.

Lieblich, A. \& Tuval-Mashiach, R. (1998). Narrative research. Londres: Sage.

Martucelli, D. (2010). iExisten individuos en el sur? Santiago de Chile: LOM Ediciones.

Mead, G. (1972). Espíritu, persona y sociedad desde el punto de vista del conductismo social. Buenos Aires: Paidós.

Nardi, H. (2006). Etica, trabalho e Subjetividade. Porto Alegre: UFRGS.

Pulido-Martínez, H. C. (2007). Produciendo trabajadores modernos. Conocimiento psicológico y el mundo del trabajo en el sur. Universitas Psychologica, 6(1), 27-38.

Ricoeur, P. (1996). Símismo como otro. Madrid: Siglo XXI.

Riessman, C. (1993). Narrative analysis. Londres: Sage.

Schvarstein, L. \& Leopold, L. (Comps.). (2005). Trabajo y subjetividad. Entre lo existente y lo necesario. Buenos Aires: Paidós.

Sennett, R. (2002). La corrosión del carácter: las consecuencias personales del trabajo en el nuevo capitalismo. Barcelona: Anagrama.

Sennett. R. (2006). La cultura del nuevo capitalismo. Barcelona: Anagrama.

Sisto, V. (2009). Cambios en el trabajo, identidad e inclusión social en Chile: desafíos para la investigación. Universum, 24(2), 192-216.

Smith, B. \& Sparkes, A. C. (2008). Contrasting perspectives on narrating selves and identities: An invitation to dialogue. Qualitative Research, 8(1), 5-35. doi:10.1177/1468794107085221

Solis, M. (2009). Trabajar y vivir en la frontera. Identidades laborales en las maquiladoras de Tijuana. Tijuana: El Colegio de la Frontera Norte.

Somers, M. R. (1994). The narrative constitution of identity: A relational and network approach. Theory and Society, 23(5), 605-649. doi: 10.1007/ BF00992905

Soto, A. (Ed.). (2008). Flexibilidad laboral y subjetividades: hacia una comprensión psicosocial del empleo contemporáneo. Santiago, Chile: LOM/Universidad Alberto Hurtado.

Spink, P. (2011). Whatever happened to work: From the centrality of shoes, ships and sealing-wax to the problems posed by flying pigs. Athenea Digital, 11(3). Extraído de http://psicologiasocial.uab.es/ athenea/index.php/atheneaDigital/article/viewFile/955/598

Stecher, A. (2010). El análisis crítico del discurso como herramienta de investigación psicosocial del mundo del trabajo: discusiones desde América Latina. Universitas Psychologica, 9(1), 93-107.

Stecher, A. (2011). Transformaciones del trabajo y procesos identitarios en el "nuevo" capitalismo: notas para una discusión en el contexto lationamericano. En B. Medrano \& W. C. Galindo (Orgs.), Psicología social e seus movimentos: 30 años da ABRAPSO (pp. 207-233). Recife: Edit Universitária da UFPE. Stecher, A. (2012). Perfiles identitarios de trabajadores de grandes empresas del retail en Santiago de Chile: aportes psicosociales a la comprensión de las identidades laborales. Psykhe, 21(2). doi:10.7764/ psykhe.21.2.538

Thompson, J. B. (1993). Ideología y cultura moderna. México: UAM.

Thompson, J. B. (1998). Los media y la modernidad. Barcelona: Paidós.

Tittoni, J. \& Nardi, E. (2011). Sujetividade e Trabalho. En L. Holzmann \& A. Cattani (Orgs.), Dicionário de trabalho e tecnología (pp. 375-378). Porto Alegre: Zouk Editora.

Wilkis, A. \& Battistini, O. (2005). El valor del trabajo en las construcciones identitarias de un grupo de asalariados jóvenes. Cuadernos de Antropología Social, 22, 55-75. 